\title{
Magnetic Properties of "As-Prepared" and Chemically Modified Multiwalled Carbon Nanotubes
}

\author{
I. Kuryliszyn-Kudelska ${ }^{a, *}$, A. MaŁolepszy ${ }^{b}$, M. MaZurkiewicz $^{b}$, L. Stobinski $^{b, c}$ \\ AND W. DOBROWOLSKI ${ }^{a}$ \\ ${ }^{a}$ Institute of Physics, Polish Academy of Sciences, al. Lotników 32/46, 02-668 Warsaw, Poland \\ ${ }^{b}$ Institute of Physical Chemistry, Polish Academy of Sciences, M. Kasprzaka 44/52, 01-224 Warsaw, Poland \\ ${ }^{c}$ Warsaw University of Technology, Faculty of Materials Science and Engineering \\ Wołoska 141, 02-507 Warsaw, Poland
}

\begin{abstract}
In this work we have studied the structural and magnetic properties of "as-prepared" multiwalled carbon nanotubes, synthesized by the chemical vapor deposition method, and chemically modified multiwalled carbon nanotubes. Transmission electron microscopy was used to show multiwalled carbon nanotubes structure. The results of systematic magnetic property measurements on multiwalled carbon nanotubes are presented. The static and dynamic magnetic responses, such as the temperature dependence of the linear AC susceptibility and DC magnetization up to $9 \mathrm{~T}$ were studied. We have observed significant changes in magnetic AC susceptibility and magnetization for "as-prepared" and chemically modified multiwalled carbon nanotubes samples.
\end{abstract}

PACS: 61.48.De, 81.20.Ym, 75.75.-c

\section{Introduction}

In recent years there has been increasing interest and development efforts in the fabricating of nanostructures, such as nanoparticles, nanowires and nanotubes due to their potential applications in electronic, magnetic or optical devices. Particularly, many serious applications have been proposed for carbon nanotubes (CNTs) [1].

To find commercial applications, CNTs are usually modified in a different way. Chemical modifications can create covalent bonding of chemical groups (such as: $-\mathrm{COOH},-\mathrm{OH},-\mathrm{NH}_{2}$, etc.) with the tube ends and CNTs walls. The chemical groups can enhance the wetting properties of CNTs in water suspensions and initiate binding interactions between matrix materials, such as polymers, metals, textiles and functionalized CNTs.

Carbon covered ferromagnetic nanowires have significant potential in data storage technology due to their size and enhanced magnetic coercivities. In addition, the carbon shells provide an effective barrier against oxidation and consequently ensure a long-term stability of ferromagnetic core. Ferromagnetic filled CNTs appear to have a significant potential in biomedical applications [2].

At present, chemical vapor deposition (CVD) method $[3,4]$, in which CNTs are synthesized in large-scale and high quality, makes more serious potential applications of CNTs. The CVD method is a promising technique be-

* corresponding author; e-mail: kuryl@ifpan.edu.pl cause it has advantages, for example, of possibility of fabrication of carbon nanotubes in large quantities, simplicity of this process and its low cost.

According to our present knowledge, carbon nanotubes do not reveal any intrinsic ferromagnetic properties at finite temperature. However, CNTs synthesis by the CVD method is performed in the presence of catalysts, such as $\mathrm{Fe}, \mathrm{Ni}$ or $\mathrm{Co}$, creating magnetic nanoparticles. For all potential applications, it is important to study the magnetic properties of carbon nanotubes produced by the CVD technique. Complete purification of "as-prepared" CNTs from the traces of iron is difficult and very often impossible. Treatment of carbon nanotubes by nitric and sulphuric acids (see e.g. [5]) is very often used. It was shown by the Mössbauer spectroscopy studies that the components characteristic for $\mathrm{Fe}_{3} \mathrm{C}, \alpha$-Fe are observed for samples prepared by CVD method using Fe as a catalyst (see e.g. [6]).

The aim of this work was to establish the method of purification of "as-prepared" MWCNTs samples produced by CVD technique. We have studied the magnetic properties of "as-prepared" and chemically purified MWCNTs. Oxidized carbon nanotubes are denoted here as MWCNTs-COOH and their ammonia salt as MWCNTs-COONH 4 .

The AC magnetic susceptibility measurements and DC magnetization studies up to $9 \mathrm{~T}$ were performed. The samples were characterized by means of transmission electron microscopy (TEM) technique. 


\section{Experimental and results}

MWCNTs (CNT Co., Ltd., Korea) were prepared by the CVD method using Fe catalyst. The "as-prepared" MWCNTs were boiled with concentrated (68\%) $\mathrm{HNO}_{3}$ under a reflux condenser for about $50 \mathrm{~h}$ at $120^{\circ} \mathrm{C}$. Amorphous carbon, traces of catalysts and their supports were gradually removed. Additionally, the raw MWCNTs material was oxidized. Carboxylic groups were attached to the ends and wall of CNTs (MWCNTs-COOH). After treatment of carboxylated MWCNTs in aqua ammonia ammonium salt we obtained $\left.\left(\mathrm{MWCNTs}_{\mathrm{COONH}}\right)\right)$. The final products were rinsed in distilled water until $\mathrm{pH}$ of filtrate was stabilized.

Figure 1 shows the transmission electron microscopy (TEM) image of the purified and oxidized MWCNTs sample. The TEM investigations show that nanotubes are very disordered and bundled. We supposed that the black spots in the picture are Fe aggregates. We may conclude that Fe nanoparticles were not completely removed during the chemical treatment. We noticed that the Fe nanoparticles were still encapsulated with graphene layers.

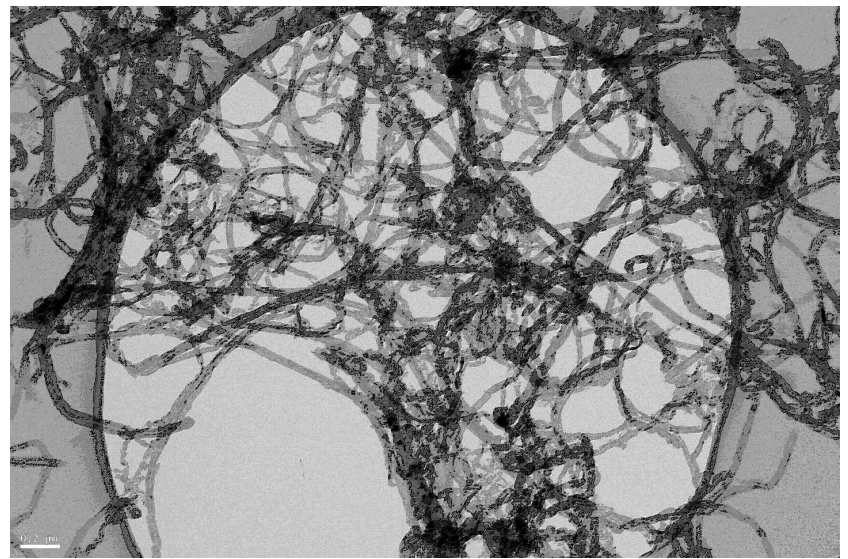

Fig. 1. TEM picture for purified MWCNTs (MWCNTs-COOH).

The systematic magnetic measurements were performed. The AC magnetic susceptibility $\chi$ studies in the temperature range $4.2-160 \mathrm{~K}$ using a mutual inductance method were carried out. Figure 2 demonstrates the temperature dependence of the real part of magnetic susceptibility $\operatorname{Re}(\chi)$ measured in the AC magnetic field of frequency $625 \mathrm{~Hz}$ and amplitude 5 Oe. We have observed the distinct decrease in magnetic susceptibility for purified MWCNTs.

For all studied samples we have observed hysteresis loops in DC magnetization versus magnetic field. Figure 3 shows the magnetization versus field curves (hysteresis loops) up to $\pm 15 \mathrm{kOe}$ for "as-prepared" and purified (oxidized) MWCNTs, measured at $4.5 \mathrm{~K}$. For all samples profiles indicate ferromagnetic behavior, which can be attributed to the Fe nanoparticles captured dur-

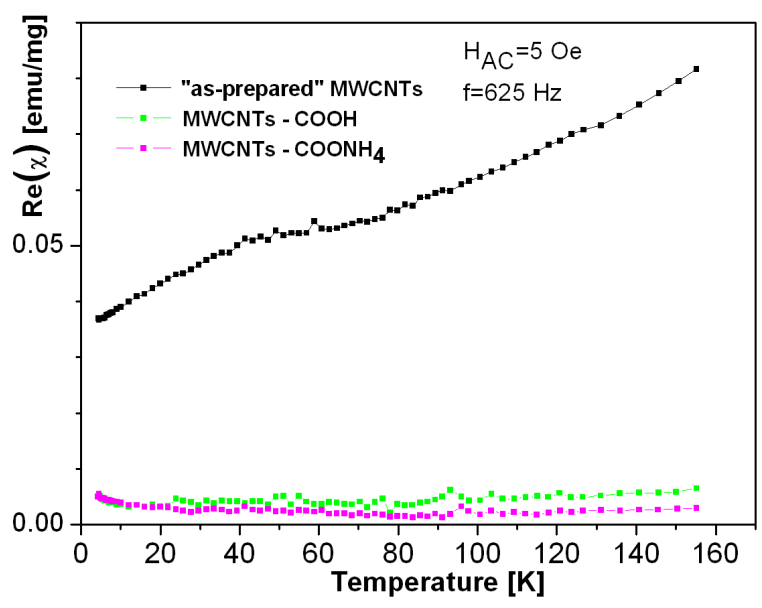

Fig. 2. The temperature dependence of the real part of magnetic AC susceptibility $\operatorname{Re}(\chi)$ for "as-prepared" MWCNTs and chemically modified MWCNTs.

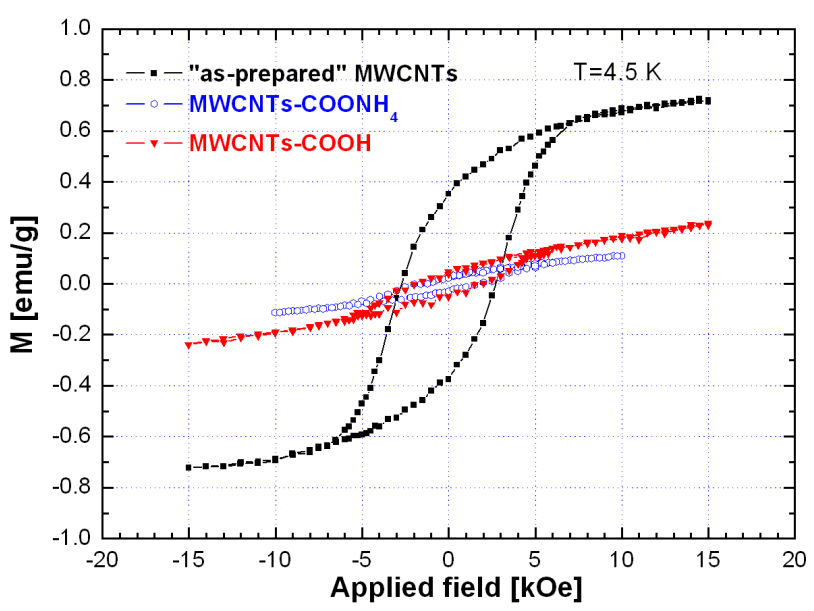

Fig. 3. Magnetic field dependence of magnetization; hysteresis loops for "as-prepared" MWCNTs, MWCNTs$-\mathrm{COOH}, \mathrm{MWCNTS}-\mathrm{COONH}_{3}$.

ing the CVD process. We have observed decrease in determined magnetic parameters values after purification processes. The value of the coercivity field $\left(H_{\mathrm{c}}\right)$ decreased from $2.78 \mathrm{kOe}$ for "as-prepared" MWCNTs to $1.78 \mathrm{kOe}$ for MWCNTs-COONH 4 and to $1.5 \mathrm{kOe}$ for MWCNTs-COOH. We have observed considerable reduction in remanent magnetization $M_{\mathrm{r}}$ from $0.36 \mathrm{emu} / \mathrm{g}$ for "as-prepared" MWCNTs to 0.03 for both purified samples.

DC magnetization measurements at high magnetic fields could help us to determine the magnetization saturation $M_{\mathrm{s}}$. Figure 4 shows magnetization versus applied magnetic field up to $9 \mathrm{~T}$ measured at $4.5 \mathrm{~K}$. It is clearly visible that the value of $M_{\mathrm{s}}$ decreased from $0.75 \mathrm{emu} / \mathrm{g}$ for "as-prepared" sample to $0.22 \mathrm{emu} / \mathrm{g}$. These values are much lower than the moments observed for bulk ferromagnetic iron (221.7 emu/g [7]) or ferromagnetic iron carbide $(160 \mathrm{emu} / \mathrm{g}[8])$. The discrepancy can be due 


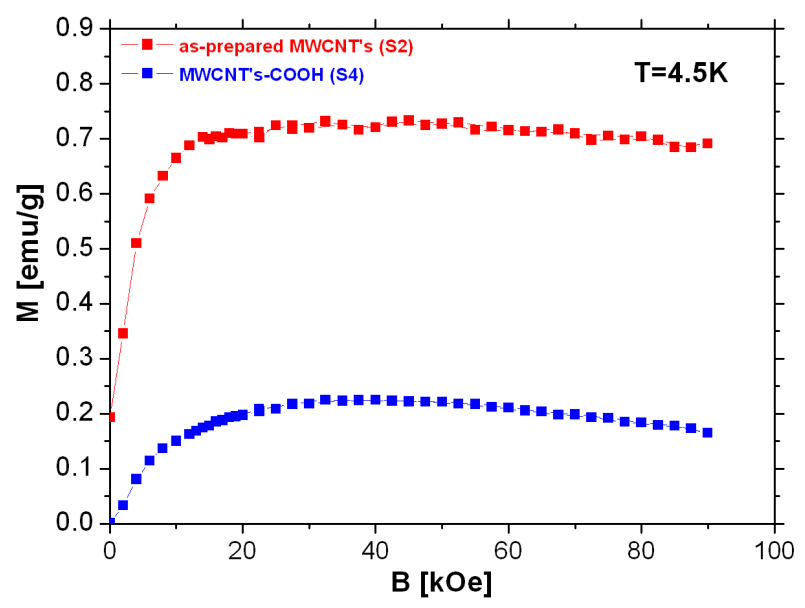

Fig. 4. High magnetic field dependence of magnetization at $4.5 \mathrm{~K}$ for "as-prepared" MWCNTs, MWCNTs$-\mathrm{COOH}$.

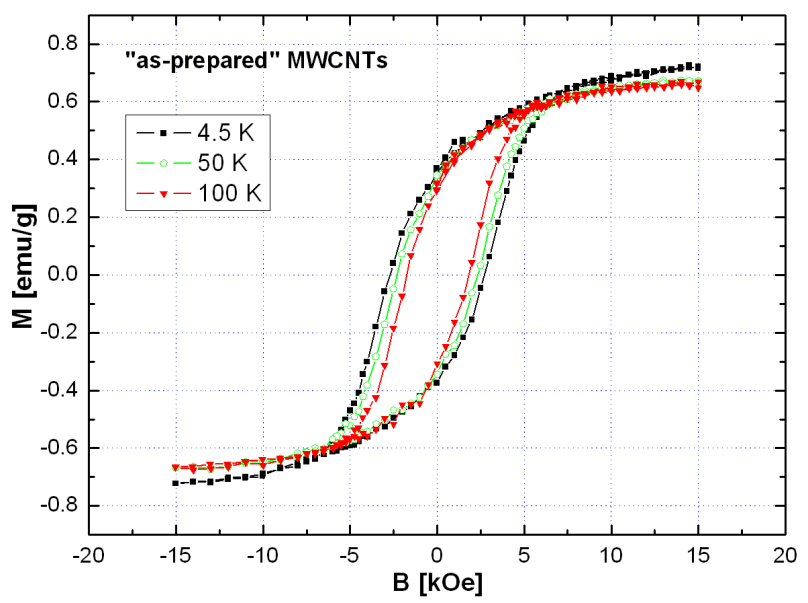

Fig. 5. Magnetic field dependence of magnetization; hysteresis loops for "as-prepared" MWCNTs obtained at $4.5 \mathrm{~K}, 50 \mathrm{~K}, 100 \mathrm{~K}$.

to the randomization of the iron nanoparticles causing a spin canting effect. The decrease in the degree of magnetization with chemical treatment indicates the removal of Fe nanoparticles that are present in CNTs due to the catalytic nanotubes formation.

A decrease in magnetization at fields $>30 \mathrm{kOe}$ was observed for both "as-prepared" and purified MWCNTs that indicates diamagnetic contribution from the graphitic material [9]. It is clearly visible that the diamagnetic influence from the graphitic nanotubes is higher for purified MWCNTs-COOH sample. It should be stressed that magnetic properties of the CVD MWCNTs are a combination of diamagnetism arising from the multiwalled nanotubes and ferromagnetism coming from iron and/or iron carbide nanoparticles. Thus, magnetization mea- surements are useful in monitoring of impact of acid treatment on both MWCNTs.

Figure 5 shows the hysteresis loops for "as-prepared" sample measured at higher temperature. The increase of $H_{\mathrm{c}}$ with decreasing temperature is clearly visible (from $1.78 \mathrm{kOe}$ for $100 \mathrm{~K}$ to $2.78 \mathrm{kOe}$ for $4.5 \mathrm{~K}$ ). Such behavior is expected and showed the anisotropic nature of the encapsulated iron and/or iron carbide nanoparticles in carbon nanotubes (see e.g. [10]).

\section{Conclusions}

We have investigated magnetic properties of the CVD multiwalled carbon nanotubes both before and after chemical treatment. We have observed decrease in the degree of magnetization with chemical treatment. AC and DC magnetic measurements revealed that chemical purification leads to notable decrease of magnetic susceptibility and coercive field, saturation magnetization. This indicates the removal of Fe nanoparticles included in CNTs due to the catalytic process. The magnetic and the TEM studies applied in this work showed that the purification process cannot completely remove the Fe contamination from CNTs.

\section{References}

[1] P.J.F. Harris, Carbon Nanotube Science: Synthesis, Properties and Applications, Cambridge Univ. Press, Cambridge (UK) 2009 (and references therein).

[2] I. Mönch, A. Meye, A. Leonhardt, K. Krämer, R. Kozhuharova, T. Gemmin, M.P. Wirth, B. Büchner, J. Magn. Magn. Mater. 276, 290 (2005).

[3] F.S. Xu, X.F. Liu, S.D. Tse, Carbon 44, 570 (2006).

[4] S. TalaPatra, S. Kar, S.K. Pal, R. Vajtai, L. Ci, P. Victor, M.M. Shaijumon, S. Kaur, O. Nalamasu, P.M. Ajayan, Nature Nanotechnol. 1, 112 (2006).

[5] S.A. Curran, A.V. Ellis, K. Vijayarahavan, P.M. Ajayan, J. Chem. Phys. 120, 4886 (2004).

[6] K. Luberda-Durnaś, M. Nieznalska, P. Tomasik, G. Khachatryan, K. Khachatryan, O. Michalski, L. Stobiński, K. Matlak, J. Korecki, K. Burda, Proc. Frontiers in Modern Physics and its Applications, Kraków 2009, p. 101.

[7] B.-X. Huang, Y.-H. Liu, J. Wang, R.-Z. Zhang, L. Zhang, L.-S. Zhang, L.-M. Mei, J. Phys., Condens. Matter. 15, 47 (2003).

[8] J.E. Hofer, E.M. Cohen, J. Am. Chem. Soc. 81, 1576 (1959).

[9] N. Grobert, W.K. Hsu, Y.Q. Zhu, J.P. Hare, H.W. Kroto, M. Terrones, P. Redlich, M. Ruhle, R. Escudero, F. Morales, Appl. Phys. Lett. 75, 3363 (1999).

[10] A.V. Eblis, B. Ingram, J. Magn. Magn. Mater. 302, 378 (2006). 